\title{
Readiness for implementation of smoke-free work hours in private companies: A qualitative study of perceptions among middle managers
}

\author{
Laerke P. Lidegaard', Maria Kristiansen², Charlotta Pisinger ${ }^{1,2,3}$
}

\begin{abstract}
INTRODUCTION Workplaces are important settings for implementation of smoke-free environments. In this study, we follow a medical production company with 677 employees that implemented one of the most stringent tobacco policies legally possible in Denmark - smoke-free work hours - which means that employees are not allowed to smoke during work time. This study explores tobacco use restrictions during work hours regarding readiness of implementation, focusing specifically on middle managers' perceptions and considerations, as well as their perceived responsibility in enforcing these.

METHODS A case study is presented. Two focus groups of 10 middle managers were conducted, seven months before smoke-free work hours were implemented. The facilitators used open-ended questions, which consisted of a structured section with specific themes related to the implementation of smoke-free work hours. Focus groups were recorded, transcribed, and analyzed thematically.

RESULTS We identified five main themes: 1) Benefits of the new policy due to better health, lower sickness-related absences, increased productivity, and improved branding value; 2) Social interactions across smoking status; 3) Smoking is a private matter with ethical dimensions; 4) The role of middle managers and concerns about enforcement; and 5) Limited experience with resistance from the smoking employees.

concLusions The findings suggest that workplaces in the preparation phase should ensure that: 1) The middle managers gain training on how to talk with employees about smoking cessation; 2) The middle managers are equipped to handle the new policy and have a joint vision and understanding of why and how they should enforce it; and 3) Room is made for discussions on resistance and enforcementrelated challenges as well as ethical issues.
\end{abstract}

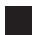 \\ AFFILIATION \\ 1 Department of Research, Danish \\ Heart Foundation, Copenhagen, \\ Denmark \\ 2 Center for Healthy Aging, \\ Department of Public Health, \\ University of Copenhagen, \\ Copenhagen, Denmark \\ 3 Center for Clinical Research \\ and Prevention, Bispebjerg- \\ Frederiksberg University Hospital, \\ University of Copenhagen, \\ Copenhagen, Denmark
}

\section{CORRESPONDENCE TO}

Lærke P. Lidegaard. Department of Research, Danish Heart Foundation, Vognmagergade 7, 3. Sal 1120 Ká benhavn K, Copenhagen, Denmark. E-mail: Ilg@hjerteforeningen.dk

\section{KEYWORDS}

smoking ban, qualitative study, smoke-free work hours, middle managers, tobacco-free workplace, readiness for implementation

Received: 3 December 2020 Revised: 23 March 2021 Accepted: 23 March 2021

\section{INTRODUCTION}

Since most adults spend about a third of their day working, workplaces provide an opportune setting for implementing smoke-free environments ${ }^{1}$. Smoking restrictions in the workplace are an important component of tobacco control policy because they protect non-smokers from the harmful health effects of passive smoking ${ }^{2}$. Furthermore, smoking restrictions provide a supportive environment for people who want to quit smoking by reinforcing social norms, and even have the potential to reduce social inequality in smoking ${ }^{2,3}$. Smoke-free workplaces encourage quitting and a reduction in smoking rates $^{3,4}$. Additionally, individuals working in smokefree environments are less likely to begin smoking than those who are exposed to smoke $e^{3,5}$.

The harmful effects of passive smoking have served as the main justifications for the smoking 
bans; other strong arguments, however, pertain to the great health benefits of not smoking ${ }^{2,6-8}$, the costs of employee's smoking such as decreased work productivity, and the need for more sick days compared to non-smoking employees ${ }^{9,10}$. Furthermore, the implementation and enforcement of health policies has been found to protect and promote mental health in the workplace ${ }^{11}$.

'The Smoke-free Environment Act', which was implemented in Denmark in 2007, banned indoor smoking in almost all public venues, including workplaces $^{12}$. The law permits smoking in oneperson offices and smoking areas, and smoking cabins are still allowed in workplaces. It also allows employees to leave indoor areas during breaks and smoke outside. In recent years, several municipalities and workplaces, both private and public, have implemented an even stricter smoking policy 'smoke-free work hours' - where an employee may not smoke at any point during his or her work time. This amount of time averages to about 7.5 hours a day despite minor differences in how breaks are placed and paid; for example, only during the self-paid lunch break is in some workplace 'legal' to smoke doing work time. Previously research primarily focused on the effectiveness of different kind of smoking or tobacco: ban, policies, legislation, restriction, law and rules at workplaces ${ }^{3-5}$, and some provided information on how these strategies were implemented ${ }^{13-17}$. The smoke-free strategy is a much stricter prevention method than former strategies that prevented smoking at most locations and not during work hours.

This study explores the organizational readiness of a middle-sized Danish medical production company to implement smoke-free work hours, seven months before the implementation. At this company, the current smoking policy bans indoor cigarette smoking. Smoking is allowed outside at three different locations at the company site. The policy furthermore allows employees to smoke during breaks.

This study contributes further insight into tobacco use restrictions during work hours by focusing on readiness for implementation among middle managers. Successful policy implementation requires substantial organizational efforts related to the commitment and support of top managers, internal communication about the project, and the organization's support activities and policy enforcement $^{18,19}$. Furthermore, middle managers are expected to play an increasingly significant role in policy implementation since they are in a unique position to potentially influence the decisions of top management as well as those of frontline employees ${ }^{18,20}$. Research that has assessed the perceptions of middle managers, related to their readiness for the implementation of smoking ban and their perceived responsibility for the implementation, is limited. Thus, the aim of this article is to explore the perceptions and readiness of middle managers in a private company that will soon begin implementing smoke-free work hours.

\section{METHODS}

\section{Study design and setting}

This study is a part of an overall evaluation of a complex smoke-free work hours intervention that investigates the implementation and outcome results of smoke-free work hours. This current study explores the initial preparation phase.

\section{The setting}

The case study focuses on a Danish medical production company with 677 employees. The headquarters are located in Copenhagen, a municipality that offers support and advice to private companies interested in implementing a stricter smoking policy.

\section{The new tobacco policy}

As outlined in the new policy, smoking or use of e-cigarettes or heated tobacco will not be allowed after 1 January 2020 anywhere at the work site during work hours, neither indoors nor outdoors. During the daily half-hour self-paid meal break, smoking will be allowed at a 'proper distance' from the site. In practice, this means that employees cannot smoke on sidewalks and roads that surround the site or in neighboring properties. Smoking in the company's clothes during the self-paid break will also not be permitted, so employees who want to smoke must change clothing. The first violation of the smoke-free policy results in a warning, and further violations may have consequences for an employee's employment. Employees have been notified of the new smoking policy one and a half years in advance. The company 
offers free smoking cessation counselling and nicotine patches.

\section{Data collection}

We adopted a qualitative approach using focus group because this methodology is suitable for generating rich and detailed information about experiences and perspectives regarding the implementation process ${ }^{21}$.

\section{Study participants}

A total of 18 middle managers were employed at the time, and 10 were recruited for the focus group; the rest of the middle managers were unavailable at the time of the focus groups. Middle managers are defined by having management responsibility for a group of employees at the company.

\section{Focus groups}

We conducted two one-hour focus groups that included three to seven participants. Focus groups took place at the company site in May 2019 (seven months before smoke-free work hours was implemented), during working hours. The focus groups were held in Danish. Two trained health consultants from the municipality collaborated in facilitating the focus groups. They used open-ended questions structured in a guide, which consisted of a structured section with specific themes related to smoke-free work hours, as well as a section that took a broader approach.

The guide was prepared by the first author in collaboration with the consultants from the municipality and revised by co-authors for content

\section{Table 1. Topics discussed during interviews}

1. The responsibility of private workplaces in relation to the health of their employees.

2. The reasons for introducing the new smoking policy.

3. Expectations for the new smoking policy.

4. Success criteria for the new smoking policy.

5. Assessment of significant barriers to the implementation of the new smoking policy.

6. Considerations on communication to employees regarding the new smoking policy.

7. Are there any expectations from top management to enforce these policies? validity. The interview guide was inspired by Weiner's theory on organizational readiness for change $\mathrm{e}^{22}$ in order to capture readiness. The facilitators encouraged interaction and discussion through open-ended questions. The topics that were discussed are shown in Table 1.

According to Danish regulations, ethical approval is not necessary for qualitative studies ${ }^{23}$. All the participants provided written informed consent. The study complied with the Declaration of Helsinki and was approved by the Danish Data Protection Agency.

\section{Statistical Analysis}

All focus groups were recorded and transcribed verbatim. We used an abductive approach in which the data were analyzed through multiple readings of the material informed by theories on organizational change. They key term related to organizational readiness was defined as 'organizational members' change commitment and change efficacy to implement organizational change based on Weiner's definition ${ }^{22}$. Data were analyzed by LPL according to Malterud's principles for systematic text condensation and guidelines for qualitative research ${ }^{24}$. First, one investigator (LPL) read the transcripts to obtain overall impressions. Second, LPL identified and coded statements or units of meaning. Third, LPL condensed and abstracted the meaning within each code that was performed and, finally, summarized the contents of the codes into primary themes. Themes were further explored and developed in collaboration with MK and CP through a critical rereading of the transcripts to identify similarities and differences among participants. Analysis resulted in identification of themes that recurred across the two focus groups. Quotations were edited for readability purposes by inserting brackets where pauses were held or where text had been left out. The Danish citations were translated into English and reviewed by all authors.

\section{RESULTS}

Ten middle managers who oversaw different types of employees (blue/white-collar) participated in the study. Seven of these were men (Table 2). The mean duration of employment was almost six years. Three were former smokers, one was a non-daily smoker, and one was a current smoker. One had been involved in the decision to implement smoke-free work hours 
Table 2. Characteristics of participants

$\begin{array}{lcc}\text { Characteristics } & n & \% \\ \text { Men } & 7 & 70.0 \\ \text { Age (years), mean (SD; range) } & 40.2 & (6.5 ; 32-52) \\ \text { Smoking status } & & \\ \text { Smoker } & 1 & 10.0 \\ \text { Non-daily smoker } & 1 & 10.0 \\ \text { Former smoker } & 3 & 30.0 \\ \text { Never smoker } & 5 & 50.0 \\ \begin{array}{l}\text { Duration of employment (years), } \\ \text { mean (SD; range) }\end{array} & 5.7 & (3.3 ; 0.33-10)\end{array}$

and another in framing the new policy. In general, there was an open and reflective dialogue during the focus groups, where the participants exchanged views. All the participants spoke.

Data analysis led to the emergence of five main themes.

\section{Theme 1: Benefits of the new smoking ban}

The first theme relates to middle managers' expectations regarding how the new smoking policy will be beneficial for both the company and the employees. All the middle managers supported the decision.

Participants emphasized that the benefits of the new smoking policy will promote better health among employees, lower sickness-related absences, increase productivity, and improve branding and image value for the company:

'And there are political signals in becoming a smoke-free business. Of course, there is. These days it is popular to be a smoke-free business.' (man, non-daily smoker)

'First off, you can say that it is in the business' own interest, really. If we are interested in our employees, for one, we get employees that are happier, but we hopefully also get employees that will attend work more days of the year due to less illness.' (man, neversmoker)

'And if they (the employees) are healthy, then it is certainly not that and their health, which affects the work they deliver at the workplace. If they are healthy, they will have a good energy and a good energy translates to a job well done.' (man, former smoker)

Furthermore, one of the participants mentioned a production-technical benefit:

'Like from a production-theoretical view, technically, smoking brings a challenge in quality for those of us making the aseptic products, as you give off more particles after smoking. So, from the pointof-view of quality products, it is actually also a benefit that we have non-smoking employees.' (man, neversmoker)

\section{Theme 2: Social interactions across smoking status}

Breaks and social interaction with colleagues were perceived as important for the mental well-being of employees. Participants were concerned that some social interactions might be lost when employees across departments and position no longer meet during smoking breaks:

'We have a task in (converting) some of the social (interactions) that used to happen in a smokingshed where you meet people from other departments.' (woman, smoker)

On the other hand, the new policy represented the potential for improved relations between smokers and non-smokers:

'Take, for example, a team of eight, four of them leave to go outside to smoke; the other four stay in the break room to drink a cup of coffee. Suddenly something unexpected happens in the production, and you need a resource person, then it is always the ones that stayed inside, you will approach.' (man, nondaily smoker)

Another middle manager supplements him:

'This might also be the reason why the teams are getting split up.' (man, former smoker)

Participants emphasized that more fairness in the break culture perhaps could lead to better relationships between smokers and non-smokers:

'Let's pick one of the teams that I have. There are the relations (divided into) smokers, and non-smokers, and I obviously expect that they will improve their relationships (across smoking status).' (man, never smoker)

This belief was shared by another middle manager, who explained why he expected that the relations would improve:

'I very much agree, as I believe that it creates wellbeing that these teams (smokers and non-smokers) will be together. If we can create a forum where they in their 
breaks actually can do something together [...], then I believe that it would contribute to even better wellbeing.' (man, former smoker)

\section{Theme 3: Smoking (at work) is a private matter} Further data analysis revealed the emergence of middle managers' underlying worries about controversy in relation to the autonomy of employees:

'But it is also a fine line, because we are playing with that line between what is the employees' own free choice, and what boundaries can we implement here. Because it is the employees' own choice to quit smoking, but we have taken that choice for them, and now they are not allowed to smoke at their workplace anymore. So, I am thinking, there is also a limit for how much we (the business) should be involved in the employees smoking cessation, as it is private and personal; it is their own choice.' (man, never-smoker)

In particular, three middle managers perceived an ethical dimension to whether or not the company can interfere with self-paid breaks. They saw it as a restriction of personal freedom.

'It is a really really fine line because the employees that are using their lunchbreak to go smoking, well, the business is not even paying for them to be there, so in actuality, they should be allowed to do whatever they want. But then, of course, you can argue that $X X$ owns the land, therefore $X X$ has a say in the matter, but it is a fine line how much should be (controlled).' (man, never-smoker)

Because self-paid breaks are not considered part of working time, it is therefore employees' own leisure time and some participants felt that the company should not interfere with it. One of the middle managers added that the new tobacco policies would affect blue-collar and white-collar employees differently:

'And for the salaried employees, where we have approximately 200 salaried employees who, what can you say, do not have a fixed working schedule, they can just come and go, so in principle they can just leave the cadaster and go to Kløvermarken (green area nearby) to smoke, as there is no-one regulating their work schedule.' (man, never-smoker)

\section{Theme 4: The role of middle managers and concerns about enforcement}

The participants shared theirs view about their own role as middle managers. Beside the two middle manager who were not involved in either the decision to implement or in framing the new policy, the participants demand guidelines for 'how to understand and interpret the new policies':

'It is about having guidelines in advance, and having offered the right courses, so that people know in advance what we are going to do.' (man, formers smoker)

This may indicate that the middle managers need clear guidelines for actions in case of violation. Furthermore, the middle manager requires more training on how to talk with employees about smoking cessation and violation:

'Yeah, I am also thinking that (because we are) leaders, I do not feel that well prepared to help right now. And I kind of wish there were better preparation for the leaders than have a conversation with your employee, and we will offer a course for smoking cessation.' (woman, never-smoker)

'( I don't know) how to handle this. Now I am working with solely salaried employees, so they leave the site for half an hour every now and then. How do you make some fair agreements with them, if they have no intention to quit smoking, and not during work hours either? (As far as figuring) out what to do as a leader, I do not feel well prepared for that.' (woman, never-smoker)

The participants emphasize that it will be almost impossible for the employees working in the production to maintain their smoking lifestyle, whereas the employees who work in the administration departments can flex out and leave the company site to smoke:

'But also, because now we should probably really start to talk to the employees, those of yours that are smokers, about what their plans are and how we can make this thing work. Because I worry a about it. As a leader, how are you gonna handle it, especially if I was actually not aware, had not closely read that you have to go that far away, how the hell are you gonna handle it, if they are not planning to quit smoking? How do you handle the work - like the everyday life, right?' (woman, never-smoker)

The middle managers verbalized that it is important but also difficult to give support to employees who smoke and at the same time play a controlling part in tobacco policy They were therefore largely in favor of postponing the difficult 
conversations with the smoking employees.

\section{Theme 5: Limited experience with resistance from the smoking employees}

In general, the middle managers perceived that the smokers would accept the new polices and refrain from resisting changes. They stated that some of the employees who smoke actually wanted to quit smoking.

'But I kind of do not feel like we have (been) met like (with) that much dissatisfaction about it, because people have been warned so much in advance that we also somehow expect it to be complied with.' (man, former smoker)

'There are quite a lot (of employees) ... (who have) communicate [d] back that (they are fine) with that settled date, because it is still far away. So, they actually find it nice, because they want help with this, they want to stop. But they are also saying, "but when we get to October, November, December, it is going to be hard, but when we get to the first of January, it is almost impossible" but then we got to figure out how we are getting through it. Because it is my impression that they actually really want to, the majority of them, want to stop now.' (man, never-smoker)

In summary, the middle managers in this study perceived that the implementation of smokefree work hours would likely be beneficial for the company and for social interaction. However, they have concerns about the ethical dimensions of the policy, although they have not yet experienced any resistance from the smoking employees. Additionally, some considerations of their roles as middle manager in relation to their responsibilities in enforcing smoke-free work hours also emerged.

\section{DISCUSSION}

Our qualitative study explores middle managers' perceptions and concerns prior to the implementation of smoke-free work hours at their workplace. The present study showed that the middle managers support the implementation of smoke-free work hours, even though they have some concerns. Overall, they find a strict smoke-free policy beneficial for the improvement of employees' health, and in its ability to possibly lower sickness-related absences, increase productivity, and promote a positive branding and image value for the company. This is in line with evidence in the field from other studies ${ }^{2,6-10}$ and is similar to what has been identified in research among both employees and employers ${ }^{25,26}$.

In this study, the middle managers drew attention to the potential beneficial impact of improved relations between smokers and non-smokers, as a result of a fairer break culture. A previous crosssectional survey supporting this hypothesis found that the conflicts between smokers and non-smokers decreased after the implementation of a restrictive smoking policy ${ }^{27}$.

With regard to the third theme, which focused on whether or not smoking at work is a private matter, the middle managers expressed worries about the autonomy of employees, especially in regard to how the company may interfere with self-paid breaks. They argued that a comprehensive smoking ban would deprive the smokers of their rights of freedom, self-determination, and privacy. This finding is in line with a study ${ }^{28}$, which revealed similar beliefs from employers about employees' rights to smoke at work, as well as the perceptions that has been identified among employees ${ }^{29,30}$. The participants in this study did not refer to the right of non-smokers to be protected from exposure to secondhand smoke, which might be because smoking is only allowed in outdoor areas and therefore they do not feel exposed to tobacco smoke.

The issue of autonomy is complex and often raises ethical dilemmas. As Sun et al. ${ }^{30}$ wrote 'smoking is rather a personal choice than a human right. The choice of a person to start smoking is his or hers'. In reality, it is not that simple; smoking is also an addiction that usually begins in childhood or adolescence, and for many smokers it is very hard to quit, even if they really want to do so. It is important to consider that the implementation of smoke-free work hours deprives the employees of the possibility of smoking during work hours but not their need and/or right for nicotine. Alcoholfree workplaces are common today, but drinking of alcohol during working hours has previously been widely accepted ${ }^{31}$. This example might be used discussing smoking policies, to explain that social norms change. It is also relevant to keep in mind that in the 1970s, the tobacco industry's original defence against restrictions on smoking in public areas invoked arguments related to 'courtesy', 'choice', and 
'freedom'32.

An essential concern in this study was the enforcement of policies. Although the middle managers supported the smoking ban, they did not consider themselves responsible for regulating other peoples' actions. These results are in line with other studies that highlight an unwillingness to take on the role of restricting tobacco-free policies ${ }^{33,34}$. Previous qualitative research has found that barriers to enforcement were related to social norms and the culture of smoking ${ }^{35}$ and create additional work and an uncomfortable policing role among managers ${ }^{34}$. A study suggest that middle managers roles could be improved by training and clear descriptions of their roles in tobacco control and with protocols for actions in case of infringements ${ }^{33}$. These findings are similar to our findings, whereas the participants in our study also demand some practical training in how to talk with employees about smoking cessation and violation. Another study found increased confidence regarding 'the difficult conversation' and argued that they would benefit from more support in the policy implementation process, which makes them more confident in enforcing rules and regulations ${ }^{36}$.

We found that the middle managers had not yet experienced any resistance from the smoking employees, and even expressed that some of the smoking employees wanted to use the introduction of smoke-free work hours as an opportunity to quit smoking. Our results showed that middle managers' commitments are limited in relations to enforcement of the policy. Middle managers are unfamiliar with this kind of policy and the intrusive approach is still rare in workplace health promotion, which may make the middle managers doubt if it is the right thing to do for the company. The introduction and implementation of very strict smoking bans in workplaces can lead to certain dilemmas related to the middle managers' fundamental values, which may ultimately lower their willingness and readiness to implement the smoking ban. Therefore, we recommend that workplaces are made aware of this situation and address the dilemmas before the smoking ban is implemented by making room for dialogue where specific resistance and enforcementrelated challenges as well as ethical issues can be discussed.

\section{Strengths and limitations}

The present study brings new perspectives on how middle managers see their own role prior to the implementation of a strict smoking ban. This study has several potential limitations. For one, the focus group was facilitated by two health consultants from the municipality and not by the first author; the first author, however, did develop the questions guide used during the focus groups. Only one individual from the focus group was a current smoker, which might not be directly transferable to a sample of middle managers that smoke. A broader sampling which included a greater number of participants that smoke may better reflected the profile of middle managers. This study was conducted seven months before the implementation of smoke-free work hours, hence there is a risk that the conceptual understanding of the policy was too abstract and speculative in the minds of the middle managers, and, consequently, that their perceptions will change closer to implementation, not to mention as time passes by following the implementation. An informational meeting was held in November 2019 for the middle managers to review the smoking policy. Furthermore, the middle managers had the opportunity to take part in a meeting about smoking cessation and workspecific challenges and issues, e.g. enforcement.

\section{CONCLUSIONS}

The introduction of smoke-free work hours requires a balancing of the interests of all members at all levels within the organization. Overall, the middle managers support and agreed on the benefits of implementing smoke-free work hours. Nonetheless, the middle managers find it problematic to interfere with employee's smoking, they did not yet experience any resistance from the smoking employees and some employees even expressed that the smoking ban would present an opportunity to quit smoking. In addition, middle managers indicated that they may require training and clear guidelines in relation to enforcing the policy. Future research focusing on the complex process of implementing smoke-free work hours in companies, and the dynamics it entails for middle managers as well as potential moral condemnation or social exclusion is needed. In terms of planning and implementing smoking bans in workplaces, the following guiding principles may be outlined: 1) The 
middle managers must gain training on how to talk with employees about smoking cessation; 2) The middle managers must be equipped to handle the new rules and stimulate a joint vision and understanding of why and how they should enforce it; and 3) Room must be made for discussions on resistance and enforcement-related challenges, as well as ethical issues.

\section{REFERENCES}

1. Workplace health promotion. World Health Organization. Accessed September 10, 2020. https://www.who.int/ occupational_health/topics/workplace/en/

2. Frazer K, Callinan JE, McHugh J, et al. Legislative smoking bans for reducing harms from secondhand smoke exposure, smoking prevalence and tobacco consumption. Cochrane Database Syst Rev. 2016;2(2):CD005992. doi:10.1002/14651858.CD005992.pub3

3. Fichtenberg CM, Glantz SA. Effect of smoke-free workplaces on smoking behaviour: systematic review. BMJ. 2002;325(7357):188. doi:10.1136/bmj.325.7357.188

4. Hopkins DP, Razi S, Leeks KD, et al. Smokefree policies to reduce tobacco use. A systematic review. Am J Prev Med. 2010;38(Suppl 2):S275-S289. doi:10.1016/j.amepre.2009.10.029

5. Mozaffarian D, Afshin A, Benowitz NL, et al. Population approaches to improve diet, physical activity, and smoking habits: a scientific statement from the American Heart Association. Circulation. 2012;126(12):1514-1563. doi:10.1161/CIR.0b013e318260a20b

6. Eisner MD, Smith AK, Blanc PD. Bartenders' respiratory health after establishment of smoke-free bars and taverns. JAMA. 1998;280(22):1909-1914. doi:10.1001/jama.280.22.1909

7. Sargent RP, Shepard RM, Glantz SA. Reduced incidence of admissions for myocardial infarction associated with public smoking ban: before and after study. BMJ. 2004;328(7446):977-980. doi:10.1136/bmj.38055.715683.55

8. Allwright S, Paul G, Greiner B, et al. Legislation for smokefree workplaces and health of bar workers in Ireland: before and after study. BMJ. 2005;331(7525):1117. doi:10.1136/bmj.38636.499225.55

9. Kirsten W. Making the link between health and productivity at the workplace--a global perspective. Ind Health. 2010;48(3):251-255. doi:10.2486/indhealth.48.251

10. Troelstra SA, Coenen P, Boot CR, Harting J, Kunst AE, van der Beek AJ. Smoking and sickness absence: a systematic review and meta-analysis. Scand J Work Environ Health. 2020;46(1):5-18. doi:10.5271/sjweh.3848

11. Mental Health and Substance Use: Mental health in the workplace. World Health Organization. Accessed October 26, 2020. http://www.who.int/mental_health/in_the_ workplace/en/
12. Ministry of the Interior and Health, Denmark. Act No 512 of 6 June 2007: Smoke-free Environments Act. June 6, 2007. Accessed March 23, 2021. https://www.dma.dk/ Vaekst/Rammevilkaar/Legislation/Acts/Act\%20on\%20 smoke-free\%20environments.pdf

13. Osinubi OY, Sinha S, Rovner E, et al. Efficacy of tobacco dependence treatment in the context of a "smoke-free grounds" worksite policy: a case study. Am J Ind Med. 2004;46(2):180-187. doi:10.1002/ajim.20020

14. Wierenga D, Engbers LH, Van Empelen P, Duijts S, Hildebrandt VH, Van Mechelen W. What is actually measured in process evaluations for worksite health promotion programs: a systematic review. BMC Public Health. 2013;13:1190. doi:10.1186/1471-2458-13-1190

15. Mlinarić M, Hoffmann L, Kunst AE, et al. Explaining Mechanisms That Influence Smoke-Free Implementation at the Local Level: A Realist Review of Smoking Bans. Nicotine Tob Res. 2019;21(12):1609-1620. doi:10.1093/ntr/nty206

16. Ruscitti LE, Castellani F, La Torre G, De Giusti M, Dominici F, Valente P. Smoking at the workplaces in Italy after the smoking ban in the Lazio Region. Med Lav. 2021;112(1):44-57. doi:10.23749/mdl.v112i1.8779

17. Leal IM, Chen TA, Correa-Fernández V, et al. Adapting and Evaluating Implementation of a Tobacco-Free Workplace Program in Behavioral Health Centers. Am J Health Behav. 2020;44(6):820-839. doi:10.5993/AJHB.44.6.7

18. Birken SA, Lee SY, Weiner BJ, Chin MH, Chiu M, Schaefer CT. From strategy to action: how top managers' support increases middle managers' commitment to innovation implementation in health care organizations. Health Care Manage Rev. 2015;40(2):159-168. doi:10.1097/HMR.0000000000000018

19. Eriksson A, Axelsson R, Axelsson SB. Health promoting leadership - different views of the concept. Work. 2011;40(1):75-84. doi:10.3233/WOR-2011-1208

20. Birken SA, Lee SY, Weiner BJ. Uncovering middle managers' role in healthcare innovation implementation. Implement Sci. 2012;7:28. doi:10.1186/1748-5908-7-28

21. Liamputtong P. Focus Group Methodology: Principles and Practice. Sage; 2011.

22. Weiner BJ. A theory of organizational readiness for change. Implement Sci. 2009;4:67. doi:10.1186/1748-5908-4-67

23. What to notify? National Committee on Health Research Ethics. February 21, 2019. Accessed February 27, 2021. https://en.nvk.dk/how-to-notify/what-to-notify

24. Malterud K. Qualitative research: standards, challenges, and guidelines. Lancet. 2001;358(9280):483-488. doi:10.1016/S0140-6736(01)05627-6

25. Halpern MT, Taylor H. Employee and employer support for workplace-based smoking cessation: results from an international survey. J Occup Health. 2010;52(6):375382. doi:10.1539/joh.110075

26. Kava CM, Parker EA, Baquero B, et al. A qualitative assessment of the smoking policies and cessation 
activities at smaller workplaces. BMC Public Health. 2018;18(1):1094. doi:10.1186/s12889-018-6001-9

27. Gottlieb NH, Eriksen MP, Lovato CY, Weinstein RP, Green LW. Impact of a restrictive work site smoking policy on smoking behavior, attitudes, and norms. J Occup Med. 1990;32(1):1623. doi:10.1097/00043764-199001000-00008

28. Sherriff NS, Coleman L. Understanding the needs of smokers who work as routine and manual workers on building sites: results from a qualitative study on workplace smoking cessation. Public Health. 2013;127(2):125-133. doi:10.1016/j.puhe.2012.10.002

29. Pursell L, Allwright S, O'Donovan D, et al. Before and after study of bar workers' perceptions of the impact of smokefree workplace legislation in the Republic of Ireland. BMC Public Health. 2007;7:131. doi:10.1186/1471-2458-7-131

30. Sun MC, Rathoa L. Innovative call emerging from a qualitative study for workplace designated stopsmoking area. Tob Prev Cessat. 2020;6(February):1-10. doi:10.18332/tpc/115032

31. Frone MR. Prevalence and distribution of alcohol use and impairment in the workplace: a U.S. national survey. J Stud Alcohol. 2006;67(1):147-156. doi:10.15288/jsa.2006.67.147

32. Dearlove JV, Bialous SA, Glantz SA. Tobacco industry manipulation of the hospitality industry to maintain smoking in public places. Tob Control. 2002;11(2):94104. doi:10.1136/tc.11.2.94

33. Martínez C, Ballbè M, Vilardell M, Fu M, Fernández E. The role of middle managers in tobacco control after a national smoke-free hospital campus ban. BMC Health Serv Res. 2016;16(1):517. doi:10.1186/s12913-016-1764-0

34. Schultz AS, Bottorff JL, Johnson JL. An ethnographic study of tobacco control in hospital settings. Tob Control. 2006;15(4):317-322. doi:10.1136/tc.2005.015388

35. Eadie D, Heim D, Macaskill S, Ross A, Hastings G, Davies J. A qualitative analysis of compliance with smoke-free legislation in community bars in Scotland: implications for public health. Addiction. 2008;103(6):1019-1026. doi:10.1111/j.1360-0443.2008.02217.x

36. Rafi J, Ivanova E, Rozental A, Lindfors P, Carlbring P. Participants' Experiences of a Workplace-Oriented Problem Gambling Prevention Program for Managers and HR Officers: A Qualitative Study. Front Psychol. 2019;10:1494. doi:10.3389/fpsyg.2019.01494
ACKNOWLEDGEMENTS

The authors would like to especially thank the company's middle managers for participating in the study. The authors also wish to thank the municipality of Copenhagen and especially the two health consultants, Mette Grønfeldt and Annette Sylvestersen.

\section{CONFLICTS OF INTEREST}

The authors have each completed and submitted an ICMJE form for disclosure of potential conflicts of interest. The authors declare that they have no competing interests, financial or otherwise, related to the current work. L. P. Lidegaard and C. Pisinger report funding from the Danish Heart Association during the conduct of the study. M. Kristiansen reports funding from Nordea Foundation during the conduct of the study.

\section{FUNDING}

This work was supported by the Danish Heart Foundation (Grant number: 18-R126-A8734).

\section{ETHICAL APPROVAL AND INFORMED CONSENT}

According to Danish regulations, ethical approval is not necessary for qualitative studies. All the participants provided written informed consent. The study complied with the Declaration of Helsinki and was approved by the Danish Data Protection Agency.

\section{AUTHORS' CONTRIBUTIONS}

All three authors LPL, MK and CP have collaborated on research concept and design. LPL was responsible for assembly of data and for the data analysis although the final interpretation was developed in collaboration with MK and CP. LPL, MK and CP wrote the final version.

PROVENANCE AND PEER REVIEW

Not commissioned; externally peer reviewed. 